\title{
Hydrogen, the next neuroprotective agent?
}

\author{
Adrian Marchidann*,1 \\ ${ }^{1}$ Stony Brook University Hospital, State University of New York, 101 Nicolls Road, Stony Brook, NY 11794, USA \\ *Author for correspondence: Tel.: +1 631444 2599; adrian.marchidann@stonybrookmedicine.edu
}

First draft submitted: 8 February 2019; Accepted for publication: 11 April 2019; Published online: 22 August 2019

Annually, in the United States, approximately 795,000 people suffer a stroke, of which 610,000 are first attacks [1]. Treatment of acute ischemic stroke has made significant progress that involves medical or endovascular recanalization of the blocked artery [2]. In spite of this, stroke continues to be a major cause of disability and mortality. An estimated 7.2 million Americans age 20 or more report a history of stroke [1].

It has been observed that cellular damage does not end with restoration of blood flow. Although several stroke animal models have shown promising results in neuroprotection studies, all interventions in humans have failed to identify a viable molecule. Due to the complexity of the pathological processes, single molecules may not be able to stop the apoptotic and cytotoxic cascades. Additionally, the effect may be hampered by limited diffusion of the neuroprotective agent within the penumbra. Therefore, a successful drug has to be easily available, diffuse rapidly, act on multiple pathological intertwined mechanisms and have few side effects. In recent years, there has been an increasing interest in studying the neuroprotective effects of hydrogen.

The 'oxygen paradox', or reperfusion injury as it is known today, is the accelerated cell death following reperfusion of an ischemic tissue with oxygenated blood [3]. Within seconds to minutes of reperfusion, dysfunctional mitochondria produce excessive amounts of radical oxygen species (ROS), six-times higher than in normal tissue and two-times higher than during the ischemic phase $[4,5]$. Of these, hydroxyl is the most reactive, being several hundred-times stronger than hydrogen peroxide [6].

The most important ROS are singlet oxygen, hydroxyl, superoxide and peroxynitrite. They are involved in the cascade pathways off cell death and apoptosis [7]. Peroxynitrite, the result of nitric oxide reaction with superoxide anion triggers necrosis and apoptosis. It is therefore involved in inflammation, autoimmunity, atherosclerosis and neurodegenerative conditions [8].

The ROS have a high propensity for chain reaction, creating new reactive molecules. There are multiple layers of defense against ROS consisting of antioxidant molecules. Alpha-tocopherol protects the lipid membrane from peroxidation. This is further reduced by ascorbate and thiols present in cytosol. Cholesterol, plasmalogens and carotenoids interact with the more aggressive singlet oxygen and hydroxyl radical. As the highly reactive radicals have a short half-life, the location of the antioxidant molecules is important for their interception and neutralization. Singlet oxygen is transformed into superoxide by superoxide dismutase, then into hydrogen peroxide, which is further converted into water by glutathione peroxidase and catalase. Hydroxyl anion is produced from hydrogen peroxide, under the catalytic influence of iron and copper [9].

In Earth's atmosphere, hydrogen is present as an inert, colorless and odorless gas, at about $0.5 \mathrm{ppm}$. It can be produced by electrolysis of water during production of oxygen, by bacterial digestion of sugars in the gut or as a by-product of batteries. There is no evidence of toxicity due to sudden or continuous exposure other than asphyxia, if present in a concentration high enough to displace oxygen. This would occur at a concentration significantly higher than the threshold for explosion, which is $4.1 \%$ [10]. The mixture of $49 \%$ hydrogen, $50 \%$ helium and $1 \%$ oxygen (hydreliox), is used by deep-sea divers without notable toxicity [11]. Hydreliox has been used safely to diminish the neurological symptoms of high-pressure nervous syndrome in divers working at depths up to $500 \mathrm{~m}$ for $27 \mathrm{~h}[12]$.

Hydrogen has many of the required characteristics for a successful neuroprotectant: is widely available, is easy to produce, diffuses rapidly through the lipid membranes, is inert and safe to administer and reacts only with the most aggressive ROS. At the same time, it mediates multiple pathophysiologic pathways leading to apoptosis and cell death. 
In cultured cells, hydrogen protects the PC12 cell line derived from the neural crests by reducing the hydroxyl radical. At the same time, it spares other ROS which may have important physiologic roles [13]. Due to its quenching capability, hydrogen has been shown to decrease lipid peroxidation [14], prevent DNA oxidation [15] and reduce the glutamate toxicity-induced death of neurons [16].

In addition to the scavenger properties, hydrogen modulates the cellular response to oxidative stress, inflammation and apoptosis pathways. Mitophagy, the removal of dysfunctional mitochondria that produce excessive amounts of ROS is enhanced by hydrogen through the PINK1/Parkin signaling pathway [17]. Hydrogen also increases expression of the $\mathrm{Nrf2}$, which mediates the cellular response to oxidative stress via antioxidant enzymes [18]. Moreover, hydrogen attenuates the reduction of activity of the antioxidant enzymes superoxide dismutase and catalase seen in ischemic rats [19].

The anti-inflammatory effect of hydrogen is highlighted by the decrease of the number of microglia and astrocytes found in injured brain tissue $[15,20]$. Hydrogen increases the number of the regulatory $T$ cells. The level of proinflammatory cytokines such as TNF- $\alpha$, IL-6, IL-1B concentrations are decreased while the anti-inflammatory cytokines such as TGF-1 $\beta$ are increased. Finally, hydrogen activates the NF-kB that regulates many antiapoptotic factors and cellular inhibitors of apoptosis proteins [21].

Human exposure to hydrogen has been shown to be remarkably innocuous. One limiting factor of using hydrogen gas is the risk of explosion at concentrations above $4 \%$. Safer storage technologies are being developed, in particular hydride [22]. The risk of explosion can also be overcome by dissolving hydrogen in water or normal saline which can be administered orally or intravenously, respectively. Administration of lactulose which by fermentation in colon produces hydrogen is an alternative in situations that do not require a rapid administration [23].

The present challenge is translating the cellular protective mechanisms of hydrogen into clinical applications. The animal models studied include occlusion of the middle cerebral artery, common carotid arteries or four vessels occlusion and induced cardiac arrest followed by resuscitation.

In the transient focal ischemia model, administration of $2 \%$ hydrogen gas during ischemia-reperfusion or only during reperfusion reduced infarction 1 day later and behavioral deficits 7 days later [15]. Moreover, the spontaneously hypertensive stroke-prone rats who received hydrogen-rich water had fewer hemorrhagic and ischemic infarcts in cortex and hippocampus. The mechanism appears to be protection of the blood-brain barrier and suppression of matrix metalloproteinase activity in the hippocampus [24]. Following global cerebral ischemia, hydrogen increased the 7-day survival rate of mice from 8.3 to $50 \%$ [25]. Another study showed that the improvement of functional outcome after cardiac arrest is comparable to therapeutic hypothermia [26].

Human studies have also been conducted. An open-label, prospective, nonrandomized study in which 38 patients received hydrogen and edaravone showed that laboratory, ECG and radiographs of patients did not deteriorate [27]. Neutral-pH hydrogen-enriched water did not produce genetic mutations, clinical symptoms or laboratory changes. The no-observable-adverse-effect level was greater than $20 \mathrm{ml} / \mathrm{kg}$ per day. For a $60-\mathrm{kg}$ person, the equivalent amount of neutral-pH hydrogen-enriched water is at least $1.2 \mathrm{l} / \mathrm{day}$ [28].

In a clinical trial of 25 patients with cerebral ischemia, 3\% hydrogen was administered by inhalation for $1 \mathrm{~h}$ of twice daily for 7 days. Hydrogen concentration reached a plateau at $20 \mathrm{~min}$, then it decreased to $10 \%$ of the plateau after 6-18 min of cessation of administration, in arterial and venous blood, respectively. The vital signs and usual blood tests were unchanged, except for an improvement of $\mathrm{O}_{2}$ saturation in the hydrogen group, which improved clinically more rapidly than the control group $[29,30]$.

There is increasing evidence from cellular, animal and human studies suggesting that hydrogen can be administered safely as a neuroprotector during revascularization. The encouraging preliminary data call for larger randomized double-blind studies to further evaluate the role of hydrogen in the treatment of acute stroke.

\section{Financial \& competing interests disclosure}

A Marchidann serves as the principal investigator of the clinical trial Neuroprotection in Acute Ischemic Stroke (H2M) sponsored by Stony Brook University in collaboration with Korea Institute of Science and Technology, was principal investigator at SUNY Downstate for the Platelet Oriented Inhibition in New TIA and Minor Ischemic Stroke Trial (POINT) and receives publishing fees from MedLink for annual updates not related to the topic. The author has no other relevant affiliations or financial involvement with any organization or entity with a financial interest in or financial conflict with the subject matter or materials discussed in the manuscript apart from those disclosed.

No writing assistance was utilized in the production of this manuscript. 


\section{Open access}

This work is licensed under the Attribution-NonCommercial-NoDerivatives 4.0 Unported License. To view a copy of this license, visit http://creativecommons.org/licenses/by-nc-nd/4.0/

\section{References}

1. Benjamin EJ, Virani SS, Callaway CW et al. Heart disease and stroke statistics-2018 update: a report from the American Heart Association. Circulation 137(12), e67-e492 (2018).

2. Powers WJ, Rabinstein AA, Ackerson T et al. 2018 Guidelines for the early management of patients with acute ischemic stroke: a guideline for healthcare professionals from the American Heart Association/American Stroke Association. Stroke 49(3), e46-e110 (2018).

3. Hearse DJ, Humphrey SM, Chain EB. Abrupt reoxygenation of the anoxic potassium-arrested perfused rat heart: a study of myocardial enzyme release. J. Mol. Cell. Cardiol. 5(4), 395-407 (1973).

4. Armstead WM, Mirro R, Busija DW, Leffler CW. Postischemic generation of superoxide anion by newborn pig brain. Am. J. Physiol. 255(2 Pt 2), H401-H403 (1988).

5. Zweier JL, Flaherty JT, Weisfeldt ML. Direct measurement of free radical generation following reperfusion of ischemic myocardium. Proc. Natl Acad. Sci. USA 84(5), 1404-1407 (1987).

6. Setsukinai K, Urano Y, Kakinuma K, Majima HJ, Nagano T. Development of novel fluorescence probes that can reliably detect reactive oxygen species and distinguish specific species. J. Biol. Chem. 278(5), 3170-3175 (2003).

7. Kim JS, Jin Y, Lemasters JJ. Reactive oxygen species, but not Ca2+ overloading, trigger pH- and mitochondrial permeability transition-dependent death of adult rat myocytes after ischemia-reperfusion. Am. J. Physiol. Heart Circ. Physiol. 290(5), H2024-H2034 (2006).

8. Ramdial K, Franco MC, Estevez AG. Cellular mechanisms of peroxynitrite-induced neuronal death. Brain Res. Bull. 133, 4-11 (2017).

9. Sies H. Strategies of antioxidant defense. Eur. J. Biochem. 215(2), 213-219 (1993).

10. Council NR. Emergency and Continuous Exposure Guidance Levels for Selected Submarine Contaminants, Volume 2. The National Academies Press, Washington, DC, USA (2008).

11. Gardette B, Delauze HG. Techniques of underwater intervention: means, methods, research and outlook. Bull. Acad. Natl. Med. 180(5), 975-983 (1996).

12. Abraini JH, Gardette-Chauffour MC, Martinez E, Rostain JC, Lemaire C. Psychophysiological reactions in humans during an open sea dive to $500 \mathrm{~m}$ with a hydrogen-helium-oxygen mixture. J. Appl. Physiol. (1985) 76(3), 1113-1118 (1994).

13. Yu J, Yu Q, Liu Y, Zhang R, Xue L. Hydrogen gas alleviates oxygen toxicity by reducing hydroxyl radical levels in PC12 cells. PLoS ONE 12(3), e0173645 (2017).

14. Cai J, Kang Z, Liu K et al. Neuroprotective effects of hydrogen saline in neonatal hypoxia-ischemia rat model. Brain Res. 1256, 129-137 (2009).

15. Ohsawa I, Ishikawa M, Takahashi $\mathrm{K}$ et al. Hydrogen acts as a therapeutic antioxidant by selectively reducing cytotoxic oxygen radicals. Nat. Med. 13(6), 688-694 (2007).

16. Han L, Tian $\mathrm{R}$, Yan $\mathrm{H}$ et al. Hydrogen-rich water protects against ischemic brain injury in rats by regulating calcium buffering proteins. Brain Res. 1615, 129-138 (2015).

17. Wu X, Li X, Liu Y et al. Hydrogen exerts neuroprotective effects on OGD/R damaged neurons in rat hippocampal by protecting mitochondrial function via regulating mitophagy mediated by PINK1/Parkin signaling pathway. Brain Res. 1698, 89-98 (2018).

18. Zhai X, Chen X, Shi J et al. Lactulose ameliorates cerebral ischemia-reperfusion injury in rats by inducing hydrogen by activating Nrf2 expression. Free Radic. Biol. Med. 65, 731-741 (2013).

19. Ge P, Zhao J, Li S, Ding Y, Yang F, Luo Y. Inhalation of hydrogen gas attenuates cognitive impairment in transient cerebral ischemia via inhibition of oxidative stress. Neurol. Res. 34(2), 187-194 (2012).

20. Hayashida K, Sano M, Kamimura N et al. Hydrogen inhalation during normoxic resuscitation improves neurological outcome in a rat model of cardiac arrest independently of targeted temperature management. Circulation 130(24), 2173-2180 (2014).

21. Li Q, Yu P, Zeng Q et al. Neuroprotective effect of hydrogen-rich saline in global cerebral ischemia/reperfusion rats: up-regulated tregs and down-regulated miR-21, miR-210 and NF-kappaB expression. Neurochem. Res. 41(10), 2655-2665 (2016).

22. Landucci G, Tugnoli A, Cozzani V. Inherent safety key performance indicators for hydrogen storage systems. J. Hazard Mater. 159(2-3), $554-566$ (2008).

23. Chen X, Zhai X, Kang Z, Sun X. Lactulose: an effective preventive and therapeutic option for ischemic stroke by production of hydrogen. Med. Gas Res. 2, 3 (2012).

24. Takeuchi S, Nagatani K, Otani N et al. Hydrogen improves neurological function through attenuation of blood-brain barrier disruption in spontaneously hypertensive stroke-prone rats. BMC Neurosci. 16, 22 (2015).

25. Nagatani K, Wada K, Takeuchi S et al. Effect of hydrogen gas on the survival rate of mice following global cerebral ischemia. Shock 37(6), 645-652 (2012). 
26. Hayashida K, Sano M, Kamimura N et al. $\mathrm{H}(2)$ gas improves functional outcome after cardiac arrest to an extent comparable to therapeutic hypothermia in a rat model. J. Am. Heart Assoc. 1(5), e003459 (2012).

27. Nagatani K, Nawashiro H, Takeuchi S et al. Safety of intravenous administration of hydrogen-enriched fluid in patients with acute cerebral ischemia: initial clinical studies. Med. Gas Res. 3, 13 (2013).

28. Saitoh Y, Harata Y, Mizuhashi F, Nakajima M, Miwa N. Biological safety of neutral-pH hydrogen-enriched electrolyzed water upon mutagenicity, genotoxicity and subchronic oral toxicity. Toxicol. Ind. Health 26(4), 203-216 (2010).

29. Ono H, Nishijima Y, Adachi $\mathrm{N}$ et al. A basic study on molecular hydrogen (H2) inhalation in acute cerebral ischemia patients for safety check with physiological parameters and measurement of blood H2 level. Med. Gas Res. 2(1), 21 (2012).

30. Ono H, Nishijima Y, Ohta S et al. Hydrogen gas inhalation treatment in acute cerebral infarction: a randomized controlled clinical study on safety and neuroprotection. J. Stroke Cerebrovasc. Dis. 26(11), 2587-2594 (2017). 